\title{
Generalised risk-sensitive control with full and partial state observation
}

\begin{abstract}
This paper generalises the risk-sensitive cost functional by introducing noise dependent penalties on the state and control variables. The optimal control problems for the full and partial state observation are considered. Using a change of probability measure approach, explicit closed-form solutions are found in both cases. This has resulted in a new risk-sensitive regulator and filter, which are generalisations of the well-known classical results.
\end{abstract}

Keywords Risk-sensitive regulator · Risk-sensitive filter · Change of measure

\section{Introduction}

Let $(\Omega, \mathcal{F}, \mathbb{P})$ be a complete probability space, on which a $(q+p)$-dimensional standard Brownian motion $(W(t), t \geq 0)$, is defined. Consider the linear stochastic control system:

$$
\left\{\begin{array}{l}
d x(t)=[A x(t)+B u(t)] d t+C d W_{1}(t) \\
d y(t)=H x(t) d t+F^{1 / 2} d W_{2}(t) \\
x(0)=x_{0}, \quad y(0)=0
\end{array}\right.
$$

Here $W_{1}(t)$ and $W_{2}(t)$ are components of $W(t)$ of order $q$ and $p$, respectively, i.e. $W(t)=\left[W_{1}^{\prime}(t), W_{2}^{\prime}(t)\right]^{\prime}$. The initial state $x_{0}$ is a Gaussina random variable with mean $\mu_{0}$ and variance $P_{0}$, with $P_{0}$ being nonsingular. It is assumed that $x_{0}$ and $W(t)$ are independent objects. The rest of the given data are:

$$
\begin{array}{ll}
A(\cdot) \in L^{\infty}\left(0, T ; \mathbb{R}^{n \times n}\right) ; & B(\cdot) \in L^{\infty}\left(0, T ; \mathbb{R}^{n \times m}\right) ; \quad C(\cdot) \in L^{\infty}\left(0, T ; \mathbb{R}^{n \times q}\right), \\
H(\cdot) \in L^{\infty}\left(0, T ; \mathbb{R}^{p \times n}\right) ; & F(\cdot) \in L^{\infty}\left(0, T ; \mathbb{R}^{p \times p}\right), \quad \text { and } \quad F(t)>0, \quad \forall t \in[0, T] .
\end{array}
$$

Here $L^{\infty}\left(0, T ; \mathbb{R}^{n \times m}\right)$ denotes the set of $\mathbb{R}^{n \times m}$ valued uniformly bounded functions on the interval $[0, T]$. For notational simplicity, we do not indicate explicitly the time dependence of coefficients.

Address(es) of author(s) should be given 
Let $\gamma \in \mathbb{R}, \gamma \neq 0$, be given. The risk-sensitive cost functional is:

$$
J(u(\cdot))=\gamma \mathbb{E}\left\{\exp \left[\frac{\gamma}{2} x^{\prime}(T) S x(T)+\frac{\gamma}{2} \int_{0}^{T}\left[x^{\prime}(t) Q x(t)+u^{\prime}(t) R u(t)\right] d t\right]\right\} .
$$

The coefficient matrices are assumed symmetric and belong to the following spaces:

$$
Q(\cdot) \in L^{\infty}\left(0, T ; \mathbb{R}^{n \times n}\right), \quad R(\cdot) \in L^{\infty}\left(0, T ; \mathbb{R}^{m \times m}\right), \quad S \in \mathbb{R}^{n \times n},
$$

with $Q(t) \geq 0, R(t)>0, \forall t \in[0, T]$ and $S \geq 0$.

The optimal control problem of finding $u(\cdot)$ that minimises (2) subject to (1), was introduced by Jacobson in [9]. Assuming full state observation, Jacobson has given a complete solution to this problem. The optimal control is of a linear state-feedback form, and has great similarity with the linear-quadratic control [20]. An important difference, however, is that in the risk-sensitive case the optimal control depends on the intensity of noise $C(t)$, which is not the case for the linear-quadratic control. Jacobson also solves the discrete-time version of the problem and explores the relation with differential games.

After this pioneering work, several attempts were made in solving the partial observation problem by [11], [12], [16], [17]. However, it is only in [2] that the complete solution to this problem was finally obtained. The discrete-time partial observation problem was solved by Whittle in [18] (see also [19]). An important relation with robust controllers was found in [5], [6], whereas the risk-sensitive maximum principle was studied in [14], [15], [8], [10]. The optimal investment problem is particulary suitable for the application of risk-sensitive control; see for example [3], [4], [13], [7].

In this paper we introduce a certain generalisation of (2), and solve the corresponding optimal control problems in an explicit closed-form. Before we indicate this generalisation, let us first give two motivating examples. It is easy to show that

$$
\begin{aligned}
x^{\prime}(T) S x(T)= & x_{0}^{\prime} S x_{0}+\int_{0}^{T}\left\{2 x^{\prime}(t) S[A x(t)+B u(t)]+\operatorname{tr}\left(C^{\prime} S C\right)\right\} d t \\
& +\int_{0}^{T} 2 x^{\prime}(t) S C d W_{1}(t) .
\end{aligned}
$$

It is thus clear that (2), through the term $x^{\prime}(T) S x(T)$, contains a certain noise dependent penalty of the state, i.e. the term

$$
\int_{0}^{T} 2 x^{\prime}(t) S C d W_{1}(t)
$$

As a second example, consider (2) with an additional cross product term between the state and control, i.e.

$J(u(\cdot))=\gamma \mathbb{E}\left\{\exp \left[\frac{\gamma}{2} x^{\prime}(T) S x(T)+\frac{\gamma}{2} \int_{0}^{T}\left[x^{\prime}(t) Q x(t)+x^{\prime}(t) S_{1} u(t)+u^{\prime}(t) R u(t)\right] d t\right]\right\}$, 
with $S_{1} \in L^{\infty}\left(0, T ; \mathbb{R}^{n \times m}\right)$ a given matrix. Introduction of a new control variable $\rho(t) \equiv u(t)+0.5 R^{-1} S_{1}^{\prime} x(t)$, transforms this cost functional into a standard one, but with a different penalty for the state as

$$
\begin{aligned}
J(u(\cdot))= & \gamma \mathbb{E}\left\{\operatorname { e x p } \left[\frac{\gamma}{2} x^{\prime}(T) S x(T)\right.\right. \\
& \left.\left.+\frac{\gamma}{2} \int_{0}^{T}\left[x^{\prime}(t)\left(Q-0.25 S_{1} R^{-1} S_{1}^{\prime}\right) x(t)+\rho^{\prime}(t) R \rho(t)\right] d t\right]\right\} .
\end{aligned}
$$

This cost is now in a standard form, and the optimal control $\rho^{*}(t)$ can be obtained by the well-known results. From it, we find the solution to the original problem as $u^{*}(t)=\rho^{*}(t)-0.5 R^{-1} S_{1}^{\prime} x(t)$. In the case of the full observation of the state, this completely solves the problem. However, in the partial observation case, this control law can not be implemented since it depends on the state. To the best of authors knowledge, the risk-sensitive control problem with cross product term and partial observation has not been considered before, and one of the reasons may well be the above mentioned difficulty. We now show that under certain assumptions, the cost (3) can be reformulated as the one having an additional noise dependent penalty on the state. Let there exists at least one symmetric differentiable function $L:[0, T] \rightarrow \mathbb{R}^{n \times n}$, that satisfies the equation $2 L(t) B(t)+S_{1}(t)=0$, for a.e. $t \in[0, T]$. Then it is not difficult to show that $(3)$ can be written as

$$
\begin{aligned}
J(u(\cdot)) & =\gamma \mathbb{E}\left\{\operatorname { e x p } \left[\frac{\gamma}{2} x^{\prime}(T)[S-L(T)] x(T)+\frac{\gamma}{2} x_{0}^{\prime} L(0) x_{0}+\frac{\gamma}{2} \int_{0}^{T} \operatorname{tr}\left(C^{\prime} L C\right) d t\right.\right. \\
& +\frac{\gamma}{2} \int_{0}^{T}\left[x^{\prime}(t)\left(Q+\dot{L}+L A+A^{\prime} L\right) x(t)+u^{\prime}(t) R u(t)\right] d t, \\
& \left.\left.+\frac{\gamma}{2} \int_{0}^{T} 2 x^{\prime}(t) L C d W_{1}(t)\right]\right\},
\end{aligned}
$$

which contains the noise dependent penalty of the state

$$
\frac{\gamma}{2} \int_{0}^{T} 2 x^{\prime}(t) L C d W_{1}(t)
$$

Motivated by these two examples, we introduce the following generalisation of the risk-sensitive cost functional:

$$
\begin{aligned}
\bar{J}(u(\cdot)) & \equiv \gamma \mathbb{E}\left\{\operatorname { e x p } \left[\frac{\gamma}{2} x^{\prime}(T) S x(T)+\frac{\gamma}{2} \int_{0}^{T}\left[x^{\prime}(t) Q x(t)+u^{\prime}(t) R u(t)\right] d t\right.\right. \\
& \left.\left.+\frac{\gamma}{2} \int_{0}^{T}\left[x^{\prime}(t) \bar{Q}_{1}+u^{\prime}(t) \bar{R}_{1}\right] d W_{1}(t)\right]\right\},
\end{aligned}
$$

where

$$
\bar{Q}_{1}(\cdot) \in L^{\infty}\left(0, T ; \mathbb{R}^{n \times q}\right), \quad \bar{R}_{1} \in L^{\infty}\left(0, T ; \mathbb{R}^{m \times q}\right) .
$$


Here we have introduced a general noise dependent penalty of the state with the weighting matrix $\bar{Q}_{1}(t)$, which obviously contains the two earlier motivating examples as special cases. In addition, we found it natural to include a noise dependent penalty on the control with the weighting matrix $\bar{R}_{1}(t)$. This type of a cost functional appears to be new in control theory, and as such there are no general methods for solving optimal control problems based on it (e.g. dynamic programming). Therefore, we had to use a different approach to solution. It turns out that by introducing particular changes of probability measures, both the full and partial state observations problems can be transformed into standard risk sensitive control problems, from which the solution is then easily obtained. In section 2 the full state observation case is considered, whereas in section 3 the partial state observation.

\section{Full state observation}

In this section we assume that the whole state $x(t)$ of system (1) is available for measurement. Thus we consider only the state equation:

$$
\left\{\begin{array}{l}
d x(t)=[A x(t)+B u(t)] d t+C d W_{1}(t), \\
x(0)=x_{0}
\end{array}\right.
$$

Let $\mathcal{X}_{t}$ be the augmentation of $\sigma(x(s), 0 \leq s \leq t)$ by all the $P$-null sets of $\mathcal{F}$, and denote by $L_{\mathcal{X}}^{2}\left(0, T ; \mathbb{R}^{m}\right)$ the set of all $\mathcal{X}_{t}$-adapted square integrable processes. In this section we assume that $u(\cdot) \in L_{\mathcal{X}}^{2}\left(0, T ; \mathbb{R}^{m}\right)$. We wish to solve the problem:

$$
\left\{\begin{array}{l}
\min _{u(\cdot)} \bar{J}(u(\cdot)) \\
\text { s.t. }
\end{array}\right.
$$

Assumption 1 Coefficients $R$ and $\bar{R}_{1}$ are such that

$$
R+\frac{\gamma}{4} \bar{R}_{1} \bar{R}_{1}^{\prime}>0
$$

Note that due to (7), in general it is no longer necessary for $R>0$ as in the classical risk-sensitive control [9].

We introduce the matrices:

$$
\begin{aligned}
\bar{A} & \equiv A+\frac{\gamma}{2} C \bar{Q}_{1}^{\prime}-\left(B+\frac{\gamma}{2} C \bar{R}_{1}\right)\left(R+\frac{\gamma}{4} \bar{R}_{1} \bar{R}_{1}^{\prime}\right)^{-1} \frac{\gamma}{4} \bar{R}_{1} \bar{Q}_{1}^{\prime} \\
\bar{B} & \equiv B+\frac{\gamma}{2} C \bar{R}_{1}^{\prime} \\
\bar{Q} & \equiv Q+\frac{\gamma}{4} \bar{Q}_{1} \bar{Q}_{1}^{\prime}-\left(\frac{\gamma}{4}\right)^{2} \bar{Q}_{1} \bar{R}_{1}^{\prime}\left(R+\frac{\gamma}{4} \bar{R}_{1} \bar{R}_{1}^{\prime}\right)^{-1} \bar{R}_{1} \bar{Q}_{1}^{\prime}, \\
\bar{R} & \equiv R+\frac{\gamma}{4} \bar{R}_{1} \bar{R}_{1}^{\prime} .
\end{aligned}
$$


Assumption 2 The following Riccati equation has a unique global solution

$$
\left\{\begin{array}{l}
\dot{\bar{P}}+\bar{Q}+\overline{P A}+\bar{A}^{\prime} \bar{P}-\bar{P}\left(\overline{B R}^{-1} \bar{B}^{\prime}-\gamma C C^{\prime}\right) \bar{P}=0 \\
\bar{P}(T)=S
\end{array}\right.
$$

A sufficient condition for Assumption 2 to hold is: $\bar{Q} \geq 0, \overline{B R}^{-1} \bar{B}^{\prime}-\gamma C C^{\prime}>0$, $\forall t \in[0, T]$ (see, e.g. [1]). The following is the main result of this section.

Theorem 1 Let the Assumption 1 and Assumption 2 hold. Then there exists a unique solution to problem (6). The optimal control and the corresponding optimal cost are, respectively:

$$
\begin{aligned}
& \bar{u}^{*}=-\bar{R}^{-1}\left(\bar{B}^{\prime} \bar{P}+\frac{\gamma}{4} \bar{R}_{1} \bar{Q}_{1}^{\prime}\right) x(t), \\
& \bar{J}^{*}=\gamma \mathbb{E}\left[\exp \left\{\frac{\gamma}{2}\left[p(0)+x_{0}^{\prime} \bar{P}(0) x_{0}\right]\right\}\right],
\end{aligned}
$$

where $p(t)$ is the solution to the ordinary differential equation:

$$
\left\{\begin{array}{l}
\dot{p}+\operatorname{tr}\left[C^{\prime} \bar{P}(t) C\right]=0 \\
p(T)=0
\end{array}\right.
$$

Two different proof of this main result now follow. The first proof is essentially a completion of squares method, whereas the second proof is based on the idea of changing the probability measure. We include both proofs since each offers some insight into the nature of the problem by giving alternative expressions for the cost functional.

2.1 Proof of Theorem 1: the completion of squares method

Let $v(t)$ be a scalar process defined as the solution to the following stochastic differential equation:

$$
\left\{\begin{array}{l}
d v(t)=\left[u^{\prime}(t) R u(t)+x^{\prime}(t) Q x(t)\right] d t+\left[u^{\prime}(t) \bar{R}_{1}+x^{\prime}(t) \bar{Q}_{1}\right] d W_{1}(t) \\
v(0)=0
\end{array}\right.
$$

Let $H(t)$ be defined as:

$$
H(t) \equiv v(t)+x(t)^{\prime} P(t) x(t)+p(t) .
$$

where $P(t)$ is the solution to the equation

$$
\left\{\begin{array}{l}
\dot{P}+P A+A^{\prime} P+Q+\frac{\gamma}{4}\left(\bar{Q}_{1}+2 P C\right)\left(\bar{Q}_{1}+2 P C\right)^{\prime}-\left[\left(\frac{\gamma}{2}\right)^{2} \bar{R}_{1}\left(\bar{Q}_{1}^{\prime}+2 C^{\prime} P\right)+\gamma B^{\prime} P\right]^{\prime} \\
\times\left(R+\frac{\gamma}{4} \bar{R}_{1} \bar{R}_{1}^{\prime}\right)^{-1}\left[\left(\frac{\gamma}{2}\right)^{2} \bar{R}_{1}\left(\bar{Q}_{1}^{\prime}+2 C^{\prime} P\right)+\gamma B^{\prime} P\right]\left(\frac{2}{\gamma}\right)^{2} \frac{1}{4}=0 \\
P(T)=S .
\end{array}\right.
$$


It is straightforward to show that in fact $P(t)=\bar{P}(t)$, and thus the above equation has a global solution due to Assumption 2. The differential of $H(t)$ is:

$$
\begin{aligned}
d H(t) & =\left\{u^{\prime}(t) R u(t)+x^{\prime}(t) Q x(t)+x^{\prime}(t) \dot{P}(t) x(t)+2 x^{\prime}(t) P(t)[A x(t)+B u(t)]\right. \\
& \left.+\operatorname{tr}\left[C^{\prime} P(t) C\right]+\dot{p}\right\} d t+\left[u^{\prime}(t) \bar{R}_{1}+x^{\prime}(t) \bar{Q}_{1}+2 x^{\prime}(t) P(t) C\right] d W_{1}(t),
\end{aligned}
$$

Let $G(t)$ be defined as

$$
G(t) \equiv \exp [\gamma H(t) / 2] .
$$

Now it is clear that

$$
\bar{J}(u(\cdot))=\gamma \mathbb{E}[G(T)] .
$$

The differential of $G(t)$ is:

$$
\begin{aligned}
d G(t) & =\frac{\gamma}{2} G(t)\left\{u^{\prime}(t) R u(t)+x^{\prime}(t) Q x(t)+x^{\prime}(t) \dot{P} x(t)+2 x^{\prime}(t) P(t)[A x(t)+B u(t)]\right. \\
& \left.+\operatorname{tr}\left[C^{\prime} P(t) C\right]+\dot{p}\right\} d t+\frac{1}{2}\left(\frac{\gamma}{2}\right)^{2} G(t)\left[u^{\prime}(t) \bar{R}_{1}+x^{\prime}(t) \bar{Q}_{1}+2 x^{\prime}(t) P(t) C\right] \\
& \times\left[\bar{R}_{1}^{\prime} u(t)+\bar{Q}_{1}^{\prime} x(t)+2 C^{\prime} P(t) x(t)\right] d t \\
& +\frac{\gamma}{2} G(t)\left[u^{\prime}(t) \bar{R}_{1}+x^{\prime}(t) \bar{Q}_{1}+2 x^{\prime}(t) P(t) C\right] d W_{1}(t) .
\end{aligned}
$$

Due to the definition of $p(t)$, the term $\operatorname{tr}\left[C^{\prime} P(t) C\right]+\dot{p}$ in the above equation is zero. Integrating both sides of (10) and taking the expectation, gives:

$$
\begin{aligned}
& \mathbb{E}[G(T)]=\mathbb{E}[G(0)] \\
+ & \mathbb{E} \int_{0}^{T} \frac{\gamma}{2} G(t)\left\{u^{\prime}(t) R u(t)+x^{\prime}(t) Q x(t)+x^{\prime} \dot{P} x(t)+2 x^{\prime}(t) P(t)[A x(t)+B u(t)]\right\} d t \\
+ & \mathbb{E} \int_{0}^{T} \frac{1}{2}\left(\frac{\gamma}{2}\right)^{2} G(t)\left[u^{\prime}(t) \bar{R}_{1}+x^{\prime}(t) \bar{Q}_{1}+2 x^{\prime}(t) P(t) C\right]\left[R_{1}^{\prime} u(t)+Q_{1}^{\prime} x(t)+2 C^{\prime} P(t) x(t)\right] d t .
\end{aligned}
$$

We now perform the completion of squares for the terms inside the above integrals that contain $u(t)$, as follows:

$$
\begin{aligned}
& u^{\prime}(t) \frac{\gamma}{2} R u(t)+\gamma x^{\prime}(t) P(t) B u(t)+\frac{1}{2}\left(\frac{\gamma}{2}\right)^{2} u^{\prime}(t) \bar{R}_{1} \bar{R}_{1}^{\prime} u(t)+\left(\frac{\gamma}{2}\right)^{2} u^{\prime}(t) \bar{R}_{1}\left[\bar{Q}_{1}^{\prime}+2 C^{\prime} P(t)\right] x(t) \\
& =u^{\prime}(t)\left[\frac{\gamma}{2} R+\frac{1}{2}\left(\frac{\gamma}{2}\right)^{2} \bar{R}_{1} \bar{R}_{1}^{\prime}\right] u(t)+u^{\prime}(t)\left[\left(\frac{\gamma}{2}\right)^{2} \bar{R}_{1}\left[\bar{Q}_{1}^{\prime}+2 C^{\prime} P(t)\right]+\gamma B^{\prime} P(t)\right] x(t) \\
& =\left\{u(t)+\frac{1}{2}\left[\frac{\gamma}{2} R+\frac{1}{2}\left(\frac{\gamma}{2}\right)^{2} \bar{R}_{1} \bar{R}_{1}^{\prime}\right]^{-1}\left[\left(\frac{\gamma}{2}\right)^{2} \bar{R}_{1}\left(\bar{Q}_{1}^{\prime}+2 C^{\prime} P(t)\right)+\gamma B^{\prime} P(t)\right] x(t)\right\} \\
& \times\left[\frac{\gamma}{2} R+\frac{1}{2}\left(\frac{\gamma}{2}\right)^{2} \bar{R}_{1} \bar{R}_{1}^{\prime}\right] \\
& \times\left\{u(t)+\frac{1}{2}\left[\frac{\gamma}{2} R+\frac{1}{2}\left(\frac{\gamma}{2}\right)^{2} \bar{R}_{1} \bar{R}_{1}^{\prime}\right]^{-1}\left[\left(\frac{\gamma}{2}\right)^{2} \bar{R}_{1}\left(\bar{Q}_{1}^{\prime}+2 C^{\prime} P(t)\right)+\gamma B^{\prime} P(t)\right] x(t)\right\}
\end{aligned}
$$




$$
\begin{aligned}
& -x^{\prime}(t)\left[\left(\frac{\gamma}{2}\right)^{2} \bar{R}_{1}\left(\bar{Q}_{1}^{\prime}+2 C^{\prime} P(t)\right)+\gamma B^{\prime} P(t)\right]^{\prime} \\
& \times \frac{1}{4}\left[\frac{\gamma}{2} R+\frac{1}{2}\left(\frac{\gamma}{2}\right)^{2} \bar{R}_{1} \bar{R}_{1}^{\prime}\right]^{-1}\left[\left(\frac{\gamma}{2}\right)^{2} \bar{R}_{1}\left(\bar{Q}_{1}^{\prime}+2 C^{\prime} P(t)\right)+\gamma B^{\prime} P(t)\right] x(t) .
\end{aligned}
$$

The terms including the state $x(t)$, including the last term in the above equation, are:

$$
\begin{aligned}
& \frac{\gamma}{2}\left\{x^{\prime}(t) Q x(t)+x^{\prime}(t) \dot{P}(t) x(t)+x^{\prime}(t)\left[P(t) A+A^{\prime} P(t)\right] x(t)\right\} \\
& +\frac{1}{2}\left(\frac{\gamma}{2}\right)^{2} x^{\prime}(t)\left[\bar{Q}_{1}+2 P(t) C\right]\left[\bar{Q}_{1}+2 P(t) C\right]^{\prime} x(t) \\
& -x^{\prime}(t)\left[\left(\frac{\gamma}{2}\right)^{2} \bar{R}_{1}\left(\bar{Q}_{1}^{\prime}+2 C^{\prime} P(t)\right)+\gamma B^{\prime} P(t)\right]^{\prime} \\
& \times \frac{1}{4}\left[\frac{\gamma}{2} R+\frac{1}{2}\left(\frac{\gamma}{2}\right)^{2} \bar{R}_{1} \bar{R}_{1}^{\prime}\right]^{-1}\left[\left(\frac{\gamma}{2}\right)^{2} \bar{R}_{1}\left(\bar{Q}_{1}^{\prime}+2 C^{\prime} P(t)\right)+\gamma B^{\prime} P(t)\right] x(t) .
\end{aligned}
$$

Due to our assumption on $P(t)$, the whole expression (11) is zero. The cost $\bar{J}(u(\cdot))$ can now be written as:

$$
\begin{aligned}
& \bar{J}(u(\cdot))=\gamma \mathbb{E}[G(0)] \\
& +\gamma \mathbb{E} \int_{0}^{T} G(t)\left\{u(t)+\frac{1}{2}\left[\frac{\gamma}{2} R+\frac{1}{2}\left(\frac{\gamma}{2}\right)^{2} \bar{R}_{1} \bar{R}_{1}^{\prime}\right]^{-1}\left[\left(\frac{\gamma}{2}\right)^{2} \bar{R}_{1}\left(\bar{Q}_{1}^{\prime}+2 C^{\prime} P(t)\right)+\gamma B^{\prime} P(t)\right] x(t)\right\}^{\prime} \\
& \times\left[\frac{\gamma}{2} R+\frac{1}{2}\left(\frac{\gamma}{2}\right)^{2} \bar{R}_{1} \bar{R}_{1}^{\prime}\right] \\
& \times\left\{u(t)+\frac{1}{2}\left[\frac{\gamma}{2} R+\frac{1}{2}\left(\frac{\gamma}{2}\right)^{2} \bar{R}_{1} \bar{R}_{1}^{\prime}\right]^{-1}\left[\left(\frac{\gamma}{2}\right)^{2} \bar{R}_{1}\left(\bar{Q}_{1}^{\prime}+2 C^{\prime} P(t)\right)+\gamma B^{\prime} P(t)\right] x(t)\right\} d t .
\end{aligned}
$$

Since $G(t)>0$ and $R+\gamma\left(\bar{R}_{1} \bar{R}_{1}^{\prime}\right) / 4>0$, we have that for all $u(\cdot)$ the following inequality holds:

$$
\bar{J}(u(\cdot)) \geq \gamma \mathbb{E}[G(0)]
$$

This lower bound is achieved if and only if

$$
u(t)=-\frac{1}{2}\left[\frac{\gamma}{2} R+\frac{1}{2}\left(\frac{\gamma}{2}\right)^{2} \bar{R}_{1} \bar{R}_{1}^{\prime}\right]^{-1}\left[\left(\frac{\gamma}{2}\right)^{2} \bar{R}_{1}\left(\bar{Q}_{1}^{\prime}+2 C^{\prime} P(t)\right)+\gamma B^{\prime} P(t)\right] x(t)
$$

However, it is straightforward to show that this is in fact $\bar{u}^{*}(t)$ as claimed in the theorem. 
2.2 Proof of Theorem 1: the change of measure method

Here the approach is to introduce a new probability measure, under which the control problem (6) is transformed into the standard risk-sensitive control problem of Jacobson [9].

Let $\theta^{\prime}(t), Z(t)$, and $Z$ be defined as:

$$
\begin{aligned}
\theta^{\prime}(t) & \equiv-\frac{\gamma}{2}\left[x^{\prime}(t) \bar{Q}_{1}+u^{\prime}(t) \bar{R}_{1}\right] \\
Z(t) & \equiv \exp \left[-\int_{0}^{t} \theta^{\prime}(\tau) d W_{1}(\tau)-\frac{1}{2} \int_{0}^{t} \theta^{\prime}(\tau) \theta(\tau) d \tau\right], \\
Z & \equiv Z(T) .
\end{aligned}
$$

Let the new probability measure $\widetilde{\mathbb{P}}$ be defined as:

$$
\widetilde{\mathbb{P}}(\alpha) \equiv \int_{\alpha} Z(\omega) d \mathbb{P}(\omega), \quad \forall \alpha \in \mathcal{F}
$$

By Girsanov theorem, the process

$$
\widetilde{W}(t) \equiv W_{1}(t)+\int_{0}^{t} \theta(\tau) d \tau
$$

is a standard Brownian motion. Moreover, since $\mathbb{P}$ and $\widetilde{\mathbb{P}}$ are equivalent probability measures, for any $\mathcal{F}_{T}$-measurable random variable $X$, we have:

$$
\mathbb{E}[Z X]=\widetilde{\mathbb{E}}[X],
$$

where $\widetilde{\mathbb{E}}[\cdot]$ is the expectation with respect to the probability measure $\widetilde{\mathbb{P}}$. The cost functional $\bar{J}(u(\cdot))$ can now be written as:

$$
\begin{aligned}
\bar{J}(u(\cdot)) & =\gamma \mathbb{E}\left\{Z \exp \left[\frac{\gamma}{2} x^{\prime}(T) S x(T)+\frac{\gamma}{2} \int_{0}^{T}\left[x^{\prime}(t) Q x(t)+u^{\prime}(t) R u(t)\right] d t\right]\right. \\
& \left.\times \exp \left[\frac{\gamma}{2} \int_{0}^{T} \frac{\gamma}{4}\left[x^{\prime}(t) \bar{Q}_{1}+u^{\prime}(t) \bar{R}_{1}\right]\left[x^{\prime}(t) \bar{Q}_{1}+u^{\prime}(t) \bar{R}_{1}\right]^{\prime} d t\right]\right\} \\
& =\gamma \widetilde{\mathbb{E}}\left\{\exp \left[\frac{\gamma}{2} x^{\prime}(T) S x(T)+\frac{\gamma}{2} \int_{0}^{T}\left[x^{\prime}(t) Q x(t)+u^{\prime}(t) R u(t)\right] d t\right]\right. \\
& \left.\times \exp \left[\frac{\gamma}{2} \int_{0}^{T} \frac{\gamma}{4}\left[x^{\prime}(t) \bar{Q}_{1}+u^{\prime}(t) \bar{R}_{1}\right]\left[x^{\prime}(t) \bar{Q}_{1}+u^{\prime}(t) \bar{R}_{1}\right]^{\prime} d t\right]\right\} .
\end{aligned}
$$

We rearrange the terms inside the integrals as follows:

$$
\begin{aligned}
& x^{\prime}(t) Q x(t)+u^{\prime}(t) R u(t)+\frac{\gamma}{4}\left[x^{\prime}(t) \bar{Q}_{1}+u^{\prime}(t) \bar{R}_{1}\right]\left[x^{\prime}(t) \bar{Q}_{1}+u^{\prime}(t) \bar{R}_{1}\right]^{\prime} \\
= & x^{\prime}(t) Q x(t)+u^{\prime}(t) R u(t)+\frac{\gamma}{4} x^{\prime}(t) \bar{Q}_{1} \bar{Q}_{1}^{\prime} x(t)+\frac{\gamma}{4} 2 u^{\prime}(t) \bar{R}_{1} \bar{Q}_{1}^{\prime} x(t)+\frac{\gamma}{4} u^{\prime}(t) \bar{R}_{1} \bar{R}_{1}^{\prime} u(t)
\end{aligned}
$$




$$
\begin{aligned}
& =x^{\prime}(t)\left(Q+\frac{\gamma}{4} \bar{Q}_{1} \bar{Q}_{1}^{\prime}\right) x(t)+u^{\prime}(t)\left(R+\frac{\gamma}{4} \bar{R}_{1} \bar{R}_{1}^{\prime}\right) u(t)+\frac{\gamma}{2} u^{\prime}(t) \bar{R}_{1} \bar{Q}_{1}^{\prime} x(t) \\
& =x^{\prime}(t)\left[Q+\frac{\gamma}{4} \bar{Q}_{1} \bar{Q}_{1}^{\prime}-\left(\frac{\gamma}{4}\right)^{2} \bar{Q}_{1} \bar{R}_{1}^{\prime}\left(R+\frac{\gamma}{4} \bar{R}_{1} \bar{R}_{1}^{\prime}\right)^{-1} \bar{R}_{1} \bar{Q}_{1}^{\prime}\right] x(t) \\
& +\left[u(t)+\left(R+\frac{\gamma}{4} \bar{R}_{1} \bar{R}_{1}^{\prime}\right)^{-1} \frac{\gamma}{4} \bar{R}_{1} \bar{Q}_{1}^{\prime} x(t)\right]^{\prime}\left(R+\frac{\gamma}{4} \bar{R}_{1} \bar{R}_{1}^{\prime}\right) \\
& \times\left[u(t)+\left(R+\frac{\gamma}{4} \bar{R}_{1} \bar{R}_{1}^{\prime}\right)^{-1} \frac{\gamma}{4} \bar{R}_{1} \bar{Q}_{1}^{\prime} x(t)\right] \\
& =x^{\prime}(t) \bar{Q} x(t)+v^{\prime}(t) \bar{R} v(t) .
\end{aligned}
$$

Here $v(t)=u(t)+\left(R+\frac{\gamma}{4} \bar{R}_{1} \bar{R}_{1}^{\prime}\right)^{-1} \frac{\gamma}{4} \bar{R}_{1} \bar{Q}_{1}^{\prime} x(t)$. The cost functional $\bar{J}(u(\cdot))$ can thus be written as:

$$
\bar{J}(u(\cdot))=\gamma \widetilde{\mathbb{E}}\left\{\exp \left[\frac{\gamma}{2} x^{\prime}(T) S x(T)+\frac{\gamma}{2} \int_{0}^{T}\left[x^{\prime}(t) \bar{Q} x(t)+v^{\prime}(t) \bar{R} v(t)\right] d t\right]\right\}(.
$$

The state equation under the new probability measure $\widetilde{\mathbb{P}}$ and in terms of the new control variable $v(t)$ is:

$$
\begin{aligned}
d x(t) & =[A x(t)+B u(t)] d t+C d W(t) \\
& =[A x(t)+B u(t)] d t+C[d \widetilde{W}(t)-\theta(t) d t] \\
& =[A x(t)+B u(t)-C \theta(t)] d t+C d \widetilde{W}(t) \\
& =\left[A x(t)+B u(t)+C \frac{\gamma}{2} \bar{Q}_{1}^{\prime} x(t)+C \frac{\gamma}{2} \bar{R}_{1}^{\prime} u(t)\right] d t+C d \widetilde{W}(t) \\
& =\left[\left(A+\frac{\gamma}{2} C \bar{Q}_{1}^{\prime}\right) x(t)+\left(B+\frac{\gamma}{2} C \bar{R}_{1}^{\prime}\right) u(t)\right] d t+C d \widetilde{W}(t) \\
& =\left(A+\frac{\gamma}{2} C \bar{Q}_{1}^{\prime}\right) x(t) d t+\left(B+\frac{\gamma}{2} C \bar{R}_{1}^{\prime}\right)\left[v(t)-\left(R+\frac{\gamma}{4} \bar{R}_{1} \bar{R}_{1}^{\prime}\right)^{-1} \frac{\gamma}{4} \bar{R}_{1} \bar{Q}_{1}^{\prime} x(t)\right] d t \\
& +C d \widetilde{W}(t) \\
& =\left[A+\frac{\gamma}{2} C \bar{Q}_{1}^{\prime}-\left(B+\frac{\gamma}{2} C \bar{R}_{1}^{\prime}\right)\left(R+\frac{\gamma}{4} \bar{R}_{1} \bar{R}_{1}^{\prime}\right)^{-1} \frac{\gamma}{4} \bar{R}_{1} \bar{Q}_{1}^{\prime}\right] x(t) d t \\
& +\left(B+\frac{\gamma}{2} C \bar{R}_{1}^{\prime}\right) v(t) d t+C d \widetilde{W}(t) \\
& =\bar{A} x(t) d t+\bar{B} v(t) d t+C d \widetilde{W}(t) .
\end{aligned}
$$

Thus, we have transformed the original control problem into the one of minimising (12) subject to (13), which is just the standard risk-sensitive control problem [9], the solutions of which is

$$
v^{*}(t)=-\bar{R}^{-1} \bar{B}^{\prime} \bar{P} x(t)
$$

The solution to problem (6) is thus:

$$
\begin{aligned}
\bar{u}^{*}(t) & =v^{*}(t)-\left(R+\frac{\gamma}{4} \bar{R}_{1} \bar{R}_{1}^{\prime}\right)^{-1} \frac{\gamma}{4} \bar{R}_{1} \bar{Q}_{1}^{\prime} x(t), \\
& =-\bar{R}^{-1}\left(\bar{B}^{\prime} \bar{P}+\frac{\gamma}{4} \bar{R}_{1} \bar{Q}_{1}^{\prime}\right) x(t) .
\end{aligned}
$$




\section{Partial state observation}

In this section we assume that only the output $y(t)$ of (1) can be measured. We repeat (1) for convenience:

$$
\left\{\begin{array}{l}
d x(t)=[A x(t)+B u(t)] d t+C d W_{1}(t) \\
d y(t)=H x(t) d t+F^{1 / 2} d W_{2}(t) \\
x(0)=x_{0}, \quad y(0)=0
\end{array}\right.
$$

Similarly to the previous section, but different from [2], we introduce the generalised risk-sensitive cost functional with an additional noise dependent penalties on state and control variables:

$$
\begin{aligned}
\widehat{J}(u(\cdot)) & =\gamma \mathbb{E}\left\{\operatorname { e x p } \left[\frac{\gamma}{2} x^{\prime}(T) S x(T)+\frac{\gamma}{2} \int_{0}^{T}\left[x^{\prime}(t) Q x(t)+u^{\prime}(t) R u(t)\right] d t\right.\right. \\
& \left.\left.+\frac{\gamma}{2} \int_{0}^{T}\left[x^{\prime}(t) \widehat{Q}_{1}+u^{\prime}(t) \widehat{R}_{1}\right] d W(t)\right]\right\} .
\end{aligned}
$$

Here $\widehat{Q}_{1}(\cdot) \in L^{\infty}\left(0, T ; \mathbb{R}^{n \times(q+p)}\right), \widehat{R}_{1}(\cdot) \in L^{\infty}\left(0, T ; \mathbb{R}^{m \times(q+p)}\right)$, are given matrices. Thus, the noise dependent penalty in (15) is due to both the state and output noises.

Let $\mathcal{Y}_{t}=\sigma(y(s), 0 \leq s \leq t)$, and denote by $L_{\mathcal{Y}}^{2}\left(0, T ; \mathbb{R}^{p}\right)$ the set of all $\mathcal{Y}_{t^{-}}$ adapted square integrable processes. In this section we assume that $u(\cdot) \in$ $L_{\mathcal{Y}}^{2}\left(0, T ; \mathbb{R}^{p}\right)$. We are interested in solving the problem

$$
\left\{\begin{array}{l}
\min _{u(\cdot)} \widehat{J}(u(\cdot)) \\
\text { s.t. }
\end{array}\right.
$$

We solve this problem by the change of measure method introduced in the previous section, i. e. by introducing a new probability measure, we transform the original problem into an equivalent one of the type considered in [2]. However, the whole derivation is more involved now. The optimal control is found to be a linear feedback from the output of a dynamical system, which is a generalised risk-sensitive filter (state estimator).

Assumption 3 There exists at least one differentiable symmetric solution $G$ : $[0, T] \rightarrow \mathbb{R}^{n \times n}$ to the equation

$$
2 G B+\frac{\gamma}{2}\left(\widehat{Q}_{1}+\widehat{G}\right) \widehat{R}_{1}^{\prime}=0, \quad \text { a.e. } t \in[0, T],
$$

where $\widehat{G} \equiv\left[2 G C, 0_{n \times p}\right]$.

A simple example when assumption (3) holds is when $\widehat{Q}_{1} \widehat{R}_{1}^{\prime}=0$, in which case one solution to $(17)$ is $G(t)=0, \forall t \in[0, T]$. 
We introduce the following matrices:

$$
\begin{aligned}
\widehat{S} & \equiv S-G(T) \\
\widehat{Q} & \equiv Q+\dot{G}+G A+A^{\prime} G+\frac{\gamma}{4}\left(\widehat{Q}_{1}+\widehat{G}\right)\left(\widehat{Q}_{1}+\widehat{G}\right)^{\prime} \\
\widehat{R} & \equiv R+\frac{\gamma}{4} \widehat{R}_{1} \widehat{R}_{1}^{\prime}
\end{aligned}
$$

Assumption 4 The matrices $R$ and $\widehat{R}_{1}$ are such that $\widehat{R}>0$.

In the following matrix partitions, the matrices $M_{1}$ and $N_{1}$ are of dimension $q \times n$ and $q \times m$, respectively:

$$
\begin{aligned}
\frac{\gamma}{2}\left(\widehat{Q}_{1}^{\prime}+\widehat{G}^{\prime}\right) & =\left[\begin{array}{l}
M_{1} \\
M_{2}
\end{array}\right], \\
\frac{\gamma}{2} \widehat{R}_{1}^{\prime} & =\left[\begin{array}{l}
N_{1} \\
N_{2}
\end{array}\right] .
\end{aligned}
$$

We further introduce the matrices:

$$
\begin{aligned}
& \widehat{A} \equiv A+C M_{1}, \\
& \widehat{B} \equiv B+C N_{1}, \\
& \widehat{H} \equiv H+F^{1 / 2} M_{2} .
\end{aligned}
$$

Consider the following Riccati equations:

$$
\begin{gathered}
\left\{\begin{array}{l}
\dot{\widehat{P}}-\widehat{A} \widehat{P}-\widehat{P} \widehat{A}^{\prime}+\widehat{P}\left(\widehat{H}^{\prime} \widehat{F}^{-1} \widehat{H}-\gamma \widehat{Q}\right) \widehat{P}-C C^{\prime}=0 \\
\widehat{P}(0)=P_{0}
\end{array}\right. \\
\left\{\begin{array}{l}
\dot{\hat{U}}+\left(\widehat{A}^{\prime}+\gamma \widehat{Q} \widehat{P}\right) \widehat{U}+\widehat{U}(\widehat{A}+\gamma \widehat{P} \widehat{Q})-\widehat{U}\left(\widehat{B} \widehat{R}^{-1} \widehat{B}^{\prime}-\gamma \widehat{P} \widehat{H}^{\prime} F^{-1} \widehat{H} \widehat{P}\right) \widehat{U}+\widehat{Q}=0 \\
\widehat{U}(T)=\frac{1}{2}\left[(I-\gamma \widehat{S} \widehat{P}(T))^{-1} \widehat{S}+\widehat{S}(I-\gamma \widehat{P}(T) \widehat{S})^{-1}\right]
\end{array}\right.
\end{gathered}
$$

Assumption 5 There exist unique global solutions $\widehat{P}(\cdot)$ and $\widehat{U}(\cdot)$ to equations (18) and (19), respectively.

A sufficient condition for the solvability of (18) is that $\widehat{H}^{\prime} \widehat{F}^{-1} \widehat{H}-\gamma \widehat{Q}>0$, see e.g. [1]. In order to find the solution to (16), we begin with the fact that:

$$
\begin{aligned}
0 & =-x^{\prime}(T) G(T) x(T)+x_{0}^{\prime} G(0) x_{0}+\int_{0}^{T} 2 x^{\prime}(t) G C d W_{1}(t) \\
& +\int_{0}^{T}\left[x^{\prime}(t)\left(\dot{G}+G A+A^{\prime} G\right) x(t)+2 x^{\prime}(t) G B u(t)+\operatorname{tr}\left(C^{\prime} G C\right)\right] d t .
\end{aligned}
$$


The cost (15) can now be written as:

$$
\begin{aligned}
\widehat{J}(u(\cdot)) & =\gamma \mathbb{E}\left\{\operatorname { e x p } \left[\frac{\gamma}{2} x^{\prime}(T) S x(T)+\frac{\gamma}{2} \int_{0}^{T}\left[x^{\prime}(t) Q x(t)+u^{\prime}(t) R u(t)\right] d t\right.\right. \\
& +\frac{\gamma}{2} \int_{0}^{T}\left[x^{\prime}(t) \widehat{Q}_{1}+u^{\prime}(t) \widehat{R}_{1}\right] d W(t) \\
& -\frac{\gamma}{2} x^{\prime}(T) G(T) x(T)+\frac{\gamma}{2} x_{0}^{\prime} G(0) x_{0}+\frac{\gamma}{2} \int_{0}^{T} x^{\prime}(t) \widehat{G} d W(t) \\
& \left.\left.+\frac{\gamma}{2} \int_{0}^{T}\left[x^{\prime}(t)\left(\dot{G}+G A+A^{\prime} G\right) x(t)+2 x^{\prime}(t) G B u(t)+\operatorname{tr}\left(C^{\prime} G C\right)\right] d t\right]\right\}
\end{aligned}
$$

Let $\widehat{\theta}(t), \widehat{Z}(t)$, and $\widehat{Z}$ be defined as:

$$
\begin{aligned}
\widehat{\theta}^{\prime}(t) & \equiv-\frac{\gamma}{2}\left[x^{\prime}(t)\left(\widehat{Q}_{1}+\widehat{G}\right)+u^{\prime}(t) \widehat{R}_{1}\right] \\
\widehat{Z}(t) & \equiv \exp \left[-\int_{0}^{t} \widehat{\theta}^{\prime}(\tau) d W(\tau)-\frac{1}{2} \int_{0}^{t} \widehat{\theta}^{\prime}(\tau) \widehat{\theta}(\tau) d \tau\right], \\
\widehat{Z} & \equiv \widehat{Z}(T) .
\end{aligned}
$$

Let a new probability measure $\widehat{\mathbb{P}}$ be defined as:

$$
\widehat{\mathbb{P}} \equiv \int_{\alpha} \widehat{Z}(\omega) d \mathbb{P}(\omega), \quad \forall \alpha \in \mathcal{F} .
$$

By Girsanov theorem, under this measure the process

$$
\widehat{W}(t) \equiv W(t)+\int_{0}^{t} \widehat{\theta}(\tau) d \tau,
$$

is a standard Brownian motion. The explicit form of the product $\widehat{\theta}^{\prime}(t) \widehat{\theta}(t)$ is:

$$
\begin{aligned}
\widehat{\theta}^{\prime}(t) \widehat{\theta}(t) & =\left(\frac{\gamma}{2}\right)^{2}\left[x^{\prime}(t)\left(\widehat{Q}_{1}+\widehat{G}\right)+u^{\prime}(t) \widehat{R}_{1}\right]\left[\left(\widehat{Q}_{1}+\widehat{G}\right)^{\prime} x(t)+\widehat{R}_{1}^{\prime} u(t)\right] \\
& =\left(\frac{\gamma}{2}\right)^{2} x^{\prime}(t)\left(\widehat{Q}_{1}+\widehat{G}\right)\left(\widehat{Q}_{1}+\widehat{G}\right)^{\prime} x(t)+\left(\frac{\gamma}{2}\right)^{2} 2 x^{\prime}(t)\left(\widehat{Q}_{1}+\widehat{G}\right) \widehat{R}_{1}^{\prime} u(t) \\
& +\left(\frac{\gamma}{2}\right)^{2} u^{\prime}(t) \widehat{R}_{1} \widehat{R}_{1}^{\prime} u(t)
\end{aligned}
$$

The cost (20) can now be written as

$$
\begin{aligned}
\widehat{J}(u(\cdot)) & =\beta \gamma \mathbb{E}\left\{\exp \left[\frac{\gamma}{2} x^{\prime}(T) \widehat{S} x(T)+\frac{\gamma}{2} \int_{0}^{T}\left[x^{\prime}(t) \widehat{Q} x(t)+u^{\prime}(t) \widehat{R} u(t)\right] d t\right] \widehat{Z}\right\} \\
& =\beta \gamma \widehat{\mathbb{E}}\left\{\exp \left[\frac{\gamma}{2} x^{\prime}(T) \widehat{S} x(T)+\frac{\gamma}{2} \int_{0}^{T}\left[x^{\prime}(t) \widehat{Q} x(t)+u^{\prime}(t) \widehat{R} u(t)\right] d t\right]\right\},
\end{aligned}
$$




$$
\begin{aligned}
I(u(\cdot)) & \equiv \frac{\widehat{J}(u(\cdot))}{\beta} \\
& =\gamma \widehat{\mathbb{E}}\left\{\exp \left[\frac{\gamma}{2} x^{\prime}(T) \widehat{S} x(T)+\frac{\gamma}{2} \int_{0}^{T}\left[x^{\prime}(t) \widehat{Q} x(t)+u^{\prime}(t) \widehat{R} u(t)\right] d t\right]\right\},
\end{aligned}
$$

where

$$
\beta \equiv \mathbb{E}\left\{\exp \left[\frac{\gamma}{2} x_{0}^{\prime} G(0) x_{0}+\frac{\gamma}{2} \int_{0}^{T} \operatorname{tr}\left(C^{\prime} G C\right) d t\right]\right\}
$$

We now focus on writing the equations (14) under the new probability measure $\widehat{\mathbb{P}}$. Note that

$$
\widehat{\theta}(t)=-\left[\begin{array}{l}
M_{1} x(t)+N_{1} u(t) \\
M_{2} x(t)+N_{2} u(t)
\end{array}\right] .
$$

By splitting the vector $\widehat{W}(t)$ in two parts

$$
\widehat{W}(t)=\left[\begin{array}{l}
\widehat{W}_{1}(t) \\
\widehat{W}_{2}(t)
\end{array}\right],
$$

where $\widehat{W}_{1}(t)$ is an $q$-dimensional vector, we can write (14) as

$$
\left\{\begin{array}{l}
d x(t)=[A x(t)+B u(t)] d t+\left[C M_{1} x(t)+C N_{1} u(t)\right] d t+C d \widehat{W}_{1}(t) \\
d y(t)=H x(t) d t+\left[F^{1 / 2} M_{2} x(t)+F^{1 / 2} N_{2} u(t)\right] d t+F^{1 / 2} d \widehat{W}_{2}(t) \\
x(0)=x_{0}, \quad y(0)=0
\end{array}\right.
$$

We introduce an $\mathcal{Y}_{t}$-adapted process $\widehat{y}(t)$ as:

$$
\widehat{y}(t) \equiv y(t)-\int_{0}^{t} F^{1 / 2} N_{2} u(\tau) d \tau .
$$

Equations (21) can now be written in the form

$$
\left\{\begin{array}{l}
d x(t)=[\widehat{A} x(t)+\widehat{B} u(t)] d t+C d \widehat{W}_{1}(t) \\
d \widehat{y}(t)=\widehat{H} x(t) d t+F^{1 / 2} d \widehat{W}_{2}(t) \\
x(0)=x_{0}, \quad \widehat{y}(0)=0 .
\end{array}\right.
$$

The risk-sensitive control problem with partial state observation

$$
\left\{\begin{array}{l}
\min _{u(\cdot)} I(u(\cdot)), \\
\text { s.t. }(23),
\end{array}\right.
$$

is of the type considered in [2], where some sufficient conditions for the existence of a unique solution are also given. Rather than repeating such conditions here, we make the following weaker assumption. 
Assumption 6 The standard risk-sensitive control problem with partial state observation (24) has a unique solution.

It is clear that the control problems (16) and (24) are equivalent. Since the solution to (24) is given in [2], we have proved the following result.

Theorem 2 Let the assumptions Assumption 3, Assumption 4, Assumption 5, and Assumption 6 hold. Then there exist a unique solution to problem (16) given by

$$
\widehat{u}^{*}(t)=\widehat{R}^{-1} \widehat{B}^{\prime} \widehat{U} \widehat{r}(t),
$$

where $r(t)$ is provided by the state estimator

$\left\{\begin{array}{l}d \widehat{r}(t)=\left(\widehat{A}-\widehat{P} \widehat{H}^{\prime} F^{-1} \widehat{H}+\gamma \widehat{P} \widehat{Q}\right) \widehat{r}(t) d t+\left(\widehat{B}-\widehat{P} \widehat{H} \widehat{F}^{-1 / 2} N_{2}\right) u(t) d t+\widehat{P} \widehat{H}^{\prime} F^{-1} d y(t), \\ \widehat{r}(0)=\mu_{0} .\end{array}\right.$

The optimal cost in this case is

$\widehat{J}^{*}=\beta \gamma \exp \left[\frac{\gamma}{2} \mu_{0}^{\prime} \widehat{U}(0) \mu_{0}+\frac{\gamma}{2} \int_{0}^{T} \operatorname{tr}\left(\widehat{P} \widehat{Q}+\widehat{U} \widehat{P} \widehat{H}^{\prime} F^{-1} \widehat{H} \widehat{P}\right) d t\right]|[I-\gamma \widehat{S} \widehat{P}(T)]|^{-1 / 2}$

\section{Conclusions}

We have introduced a generalisation of the risk-sensitive cost functional consisting of noise dependent penalities of the state and control variables. Using the completion of squares and the change of probability measure methods, we have obtained explicit solutions to optimal control problems for the full and partial state observation. This has resulted in a more general risk-sensitive regulator and filter. It will be interesting to extend these ideas to discrete-time setting, and explore the relation with robust controllers based on $H_{\infty}$ control theory (as in $[5],[6]$ ).

\section{References}

1. Anderson, B. D. O., and Moore, J., Optimal control: linear quadratic methods, Pretice Hall, (1990).

2. Bensoussan, A., and Van Schuppen, J. H., Optimal control of partially observable stochastic systems with an exponential-of-integral performance index, SIAM J. Control and Optimisation, 23, 599-613 (1985).

3. Bielecki, T. R., Pliska, S. R., and Sheu, S. J., Risk sensitive portfolio management with Cox-Ingersoll-Ross interest rates: the HJB equation, SIAM J. Control and Optimization, 44, 1811-1843 (2005).

4. Davis, M. H. A., and Lleo, S., Jump-Diffusion Risk-Sensitive Asset Management I: Diffusion factor model, SIAM J. Fin. Math., 2, 22-54 (2011).

5. Glover, K., anf J. Doyle, State space formulae for all stabilizing controllers that satisfy an $H_{\infty}$ norm bound and relation to risk-sensitivity, Systems \& Control Letters, 11, 167-172, (1988).

6. Glover, K., Minimum entropy and risk-sensitive control: the continuous time case, Proceedings of the 28th IEEE Conference on Decision and Control, 388-391, 1989. 
7. Goel, M., and Kumar, K. S., Risk-sensitive portfolio optimization problems with fixed income securities, Journal of Optimization Theory and Applications, 142, 1, 67-84 (2009).

8. Huang, J., Li, X., and Wang, G., Maximum Principles for a Class of Partial Information Risk-Sensitive Optimal Controls, IEEE Trans. Automatic Control, 55, 6, 1438-1443, (2010).

9. Jacobson, D. H., Optimal stochastic linear systems with exponential criteria and their relation to deterministic differential games, IEEE Trans. Automatic Control, AC-18, 124131 (1973).

10. Jingtao, S, anf Zhen, W., A risk sensitive stochastic maximum principle for optimal control of jump diffusions and its applications, Acta Mathematica Scientia, 31B(2), 419 433 (2011).

11. Kraniak, J. C., Machell, F. M., Markus, S. I., and Spreyer, J. L., The dynamic linear expoential gaussian team problem, IEEE Trans. Automatic Control, 27, 860-869, (1982).

12. Kumar, P. R., and van Schuppen, J. H., On the optimal control of stochastic systems with an exponential-of-integral performance index, J. Math. Anal. Appl., 80, 312-332, (1981).

13. Kurodaa, K., and Nagaib, H., Risk-sensitive portfolio optimization on infinite time horizon, Stochastics and Stochastic Reports, 73, 309-331 (2002).

14. Lim, A. E. B., Zhou, X. Y., and Moore, J. B., Multiple-objective risk-sensitive control and its small noise limit, Automatica, 39, 533541 (2003).

15. Lim, A. E. B., and Zhou, X. Y., A new risk-sensitive maximum principle, IEEE Trans. Automatic Control, 50, 7, 958-966 (2005).

16. Speyer, J. L., Deyst, J., and Jacobson, D. H., Optimization of stochastic linear systems with additive measurement and process noise using expoential performance criteria, IEEE Trans. Automatic Control, AC-19, 358-366, (1974).

17. Speyer, J. L., An adaptive terminal guidance scheme based on an exponential cost criterion with application to homing missile gidance, IEEE Trans. Automatic Control, AC-21, 371-375, (1976)

18. Whittle, P, Risk-sensitive linear/quadratic/Gaussian control, Advances in Applied Probability, 13, 764-777 (1981).

19. Whittle, P., Risk-senistive optimal control, Wiley, (1990).

20. Wonham, W. M., Random differential equations in control theory, In A. T. BharuchaReid Ed., Probabilistic methods in applied mathematics, Vol. 2, 132-212, Academic Press, New York, 1970. 